\title{
A Multicenter Observational Study to See the Trends of Using Different Contraceptive Agents and Its Drawbacks
}

\author{
Umme Ruman ${ }^{1}$, Mir M Rhaman², Monowara Khatun ${ }^{3}$
}

\begin{abstract}
Aim: Bangladesh is a densely populated country where approximately 1,260 people are living per square kilometer. The Ministry of Health of Bangladesh and different Non-Governmental Organizations are providing free contraceptive methods, and for its availability now, our total fertility rate (TFR) is reduced to 2.3. However, due to ignorance and reluctance, family planning utilization is yet suboptimal, especially that of long-term and irreversible methods; and thus, an unexpected, as well as unplanned pregnancy is increasing at a pace. Due to the availability of over-the-counter (OTC) abortifacient drugs like misoprostol and mifepristone (MM) kit in our country, people are consuming unsupervised not only the wrong dose but also in a wrong gestational age. Thus, unsafe abortion is highly increasing, although most of these women know about different types of regular contraceptive methods. In this study, our aim was to observe the trends of using contraception, especially the long-term methods and its drawback.

Methods and materials: The study design was accepted by the Ethical Review Committee of Combined Military Hospital (CMH) of Bogura. In total, 32,629 women were enrolled in the present study. This retrograde observational study was conducted from July 2017 to June 2019 in four hospitals/clinics-CMH of Bogura, Thengamara Mahila Shobuj Shangha (TMSS) Medical College and Hospital, Mary Stopes Clinic (MSC), and Shurjer Hashi Clinic (SHC). These centers were selected purposively as they have distinct family planning section as well as they maintain well-structured data. Different parameters were compared between 2017 and 2018. Statistical analysis was done using SPSS (version 10), and the result that is lower than or equal to 0.05 has been signified as "statistically significant."

Results: Among the different contraceptions like short-acting methods [oral contraceptive pill (OCP), condom, and injectable hormones] and long-acting methods [intrauterine contraception device (IUCD) and implant] and permanent methods (tubectomy and vasectomy), the study observed that in $\mathrm{CMH}$ of Bogura, the number of OCP and condom users have significantly increased in 2018 compared to that of 2017 , and the $p$-value is 0.047 and 0.039 , respectively. In 2018, IUCD users are significantly reduced ( $p=0.0001)$ in CMH of Bogura, compared to the previous year, whereas in other hospitals/clinics, the outcome is nonsignificant. In the case of implant users, the number is almost similar in all the centers (for TMSS, $p=0.063$; SHC, $p=0.25$; MSC, $p=0.71$ ), but surprisingly in CMH of Bogura, no users were found in both years. For the injectable users, it significantly increased in MSC $(p=0.005)$ but significantly decreased in TMSS $(p=0.043)$, whereas it slightly decreased in CMH of Bogura $(p=0.13)$ and SHC $(p=0.226)$. The use of OCP was significantly increased in MSC (0.01) and CMH of Bogura $(p=0.047)$, whereas it was significantly reduced in SHC ( $p=0.008)$. In TMSS and CMH of Bogura, the condom users were significantly increased $(p=0.035$ and $p=0.039$, respectively). In the case of tubal ligation, no change was observed in any center, whereas in the case of male sterilization, it was observed that it was absent in the three centers, but in MSC, it has increased suddenly in the second year.

The number of induced abortions after using MM kit was increased. They are admitted to hospital with excess hemorrhage and infection. Preference of these agents is due to quick action and short dosage.

Conclusion: Campaign for long-acting contraception should be emphasized. Well-trained family planning officer should be employed. OTC availability of abortifacient drugs should be restricted, and it should only be prescribed by the doctor or health provider.

Keywords: Contraception, Incomplete abortion, Long-acting reversible contraception (LARC), Misoprostol.

Journal of South Asian Federation of Obstetrics and Gynaecology (2021): 10.5005/jp-journals-10006-1908
\end{abstract}

\section{INTRODUCTION}

Bangladesh has experienced a sevenfold rise in its contraceptive prevalence rate (CPR) from 1975 to 2014. It was challenging as it was a Muslim majority country in addition to lower literacy rate, there is lower independence for women to take their own decisions in a male dominant society. ${ }^{1}$ But due to discontinuation and irregular use of contraception, almost one-third of pregnancies are accidental. ${ }^{2}$ In a country, fertility is not solely dependent on the prevalence of contraceptive use. It also depends on contraceptive use-effectiveness and user adherence. Married women in Bangladesh are having 0.7 more children than they expect. If unplanned pregnancies were avoided, the total fertility rate (TFR) would be $30 \%$ less. Unmet need for family planning also plays a role in the effectiveness of family planning programs. \begin{tabular}{l}
\hline \hline Department of Obstetrics and Gynecology, Bangladesh Armed \\
Forces, Dhaka, Bangladesh \\
${ }^{2}$ Department of Public Health, New York, United States of America \\
${ }^{3}$ Department of Obstetrics and Gynaecology, Bogura, Bangladesh \\
Corresponding Author: Lt. Col. Dr. Umme Ruman, Department of Obstet- \\
rics and Gynecology, Bangladesh Armed Forces, Dhaka, Bangladesh, \\
Phone: +01711956262, e-mail: rumman09umme@yahoo.com \\
How to cite this article: Ruman U, Rhaman MM, Khatun M. A \\
Multicenter Observational Study to See the Trends of Using Different \\
Contraceptive Agents and Its Drawbacks. J South Asian Feder Obst \\
Gynae 2021;13(3):180-184. \\
Source of support: Nil \\
Conflict of interest: None
\end{tabular}

\footnotetext{
(c) Jaypee Brothers Medical Publishers. 2021 Open Access This article is distributed under the terms of the Creative Commons Attribution 4.0 International License (https://creativecommons.org/licenses/by-nc/4.0/), which permits unrestricted use, distribution, and non-commercial reproduction in any medium, provided you give appropriate credit to the original author(s) and the source, provide a link to the Creative Commons license, and indicate if changes were made. The Creative Commons Public Domain Dedication waiver (http://creativecommons.org/publicdomain/zero/1.0/) applies to the data made available in this article, unless otherwise stated.
} 
These programs address some issues related to contraceptive method use. These include the type of method used, mixing and switching of methods, discontinuation, and switching. So, it is important to understand why still almost one-third of pregnancies are unintended. ${ }^{3}$ It has been proved that long-acting reversible contraception (LARC), which includes intrauterine contraceptive devices (IUCDs) and subdermal implants, is highly effective, and they possess many ascribe like they have few contraindications, cheap, and few follow-up after initial insertion. They also have a quick return of fertility after removal. ${ }^{4}$ The World Health Organization estimated that LARC is effective in preventing unwanted pregnancy, estimating that only one unintended pregnancy occurs among the 2,000 pregnancies after the use in the first year. ${ }^{5}$ Short-acting contraceptive methods on the other have a higher failure rate in preventing unwanted pregnancies that are 90 per 1,000 users of pills and 60 per 1,000 users of the depo medroxyprogesterone acetate (DMPA) injectable. ${ }^{6}$ Even though oral contraception and condoms are predominantly used by the couples, the use of these contraceptions are still irregular and inconsistent. The most widely used method is oral pill that is $27 \%$, DMPA is $12 \%$, barrier method is $6 \%$, and female sterilization is $5 \%{ }^{7}$ The use of any kind of LARC by a newly married couple is as low as $8 \%$. So, if LARC uptake can be increased, it may play an effective role in preventing unwanted pregnancy and thus may reduce unsafe abortion. For the declining of TFR, besides the increase in contraception use, an increase in access to maternal health services like safe abortion service [including menstrual regulation $(\mathrm{MR})]$ is also contributory. Bangladesh's government does not promote abortion as a family planning method. ${ }^{8}$ Bangladesh's national family planning program has included MR since 1979. Though there is availability for MR service, many women indent for clandestine abortion. About three-fifths of unintended pregnancies are responsible for abortion and MR. MR performed using medication is designated as menstrual regulation with medication. ${ }^{9}$ Medical abortion using a combination of drugs [mifepristone and misoprostol (MM) kit] is commonly used as it is safe, efficient, and affordable. It is an acceptable and approved method of medical termination of pregnancy (MTP) up to 9 weeks of gestation. ${ }^{10}$ This method is approved for use under the amended MTP Act. Hence, this schedule alone should be strictly maintained along with all other provisions under the Act (counseling, consent, examination, confirmation of pregnancy, prescription by a registered medical practitioner, client card). ${ }^{11} \mathrm{~A}$ known disadvantage of medical abortion is incomplete abortion. It is reported in 0.2 to 0.3 of five cases. ${ }^{12,13}$ When there is a wrong selection of patients, wrong route, wrong dose and timing, and using the drug singly rather than combination, incomplete abortion may take a bad shape and comes with complications such as sepsis and hemorrhage. Mostly, these complications occur due to prescribing the drug at the wrong gestational age (it is less effective in 7-9 weeks of pregnancies) and self-prescribed medications by the patients. ${ }^{14,15}$ Many abortifacients used in medical termination of pregnancy are nowadays available as overthe-counter (OTC) drug. They can be easily purchased without any prescription in many countries, including Bangladesh. Drug sellers also give medications like oral contraceptive pills (OCP), antibiotic analgesics, and misoprostol to women, especially for clandestine abortion. Misoprostol can definitely reduce the number of unsafe abortions, but it should be prescribed by a health professional with adequate counseling to the women. ${ }^{16}$ Otherwise it may end up with incomplete abortion, septic abortion, severe hemorrhage, and shock. Above all, most of these abortions could be prevented if they took any kind of contraception with adherence, especially LARC, which is highly effective.

Seeing this trend of contraception use, the use of unsupervised misoprostol, and increased misoprostol-induced incomplete and septic abortion, we decided to perform the trend of current use of contraception and its drawback. Here, we counted the drawback as not using any contraception or effective method that increases the chance of unintended pregnancy. To get rid of this, women are endangering their lives by taking abortifacients in inappropriate way.

\section{Methods and Materials}

\section{Study Places}

We conducted the study in four public health service facilities situated in Bogura: Combined Military Hospital (CMH) of Bogura, Thengamara Mahila Shobuj Shangha (TMSS) Medical College and Hospital, Mary Stopes Clinic (MSC), and Shurjer Hashi Clinic (SHC). The purpose for choosing these places was they maintain an organized family planning section. Then, we compared consecutive 2 years (July 2017-June 2018 and July 2018-June 2019) of data on contraception consumers, their number, any difference between these two tenures in the method consumed, number of induced abortion use after the use of MM kit, and misoprostol where indoor service is present.

\section{Study Design}

This is a retrograde observational study.

\section{Study Population}

The study population consisted of women of reproductive age (15-45 years) residing in Bogura, seeking family planning services from the above-mentioned centers. The study excluded women who were coming in for contraceptive removal. Data were collected from the registrar book recorded from July 2017 to June 2018 and July 2018 to June 2019.

\section{Sampling Procedure and Sample Size}

Current metro area population of Bogura in 2020 is 775,000, a $6.16 \%$ from 2019. It was 730,000 , a $6.57 \%$ increase from 2018 . The population growth rate of Bogura is $6.16 \%$ in the year 2020 , whereas the population growth rate of Bangladesh is $1.1 \%$. Only public health facilities, because of their uniformity in the provision of free-cost FP services, were included in the study. We selected the above four centers purposively after searching as they are maintaining a wellorganized record of contraception users. The study population was 32,629 in 2 years in the four centers, seeking contraception in this period. The sampling procedure was a randomized control trial.

\section{Ethical Clearance}

Ethical Review Committee of $\mathrm{CMH}$ of Bogura permitted ethical clearance to conduct the study.

\section{Data Collection Procedure}

At first, the family planning in charge of each center was contacted. Data were collected from the registrar book recorded from July 2017 to June 2018 and July 2018 to June 2019. One family planning welfare officer (FPWA) was the research assistant. He used to maintain the 
records of family planning in CMH of Bogura. Then, he went to TMSS, MSC, and SHC in working hour after contacting the chief family planning officer. We conducted data collection for 4 days. Then, a comparison of consecutive 2 years was made between different contraception methods utilization. The main outcome we were interested for was LARC uptake. Here we categorized implants and copper IUCDs as LARC, short-term methods were, 3-month DMPA injectable, and OCPs. We interviewed concerned gynecologists where indoor facilities were available. The number of patients admitted with abortion complications, such as hemorrhage, incomplete abortion, and septic abortion after using misoprostol and MM kit, was recorded.

\section{Statistical Analysis}

Analysis was done by SPSS 20 software.

\section{Result}

In this study, a total of 32,629 patients were included who were seeking family planning methods in four health services named $\mathrm{CMH}$ of Bogura, TMSS, SHC, and MSC, which also provide structured family planning services. The first year was from July 2017 to June 2018, and the second year was from June 2018 to June 2019.

Table 1 showed that in $\mathrm{CMH}$ of Bogura during the first year, we can see that the number of IUCD users was 14 , which was reduced significantly to one in the second year ( $p$-value, 0.0001). Implant use was nil here. Users of DMPA did not show much difference (282 and 271). Consumers of OCPs and the condom were significantly increased ( $p$-value $<0.047$ and $<0.039$, respectively). Tubectomy was reduced in the second year. Vasectomy was nil. In TMSS, IUCD and implants users have increased over the 2 years. But injectables and condom users have increased significantly ( $p$-value $<0.043$ and <0.035).The number of tubectomy was almost doubled. Vasectomy was nil.

In SHC, no differences were seen between users of IUCD, implant, and DMPA. But OCP users are significantly increased ( $p$-value $<0.008$ ) in the second year. Tubectomy did not show any difference in 2 years. Vasectomy was nil.
In MSC, IUCD and implant consumers were the same. Shortterm methods like injectables and OCP also showed a significant increase in the second year ( $p$-value $<0.005$ and $<0.01$, respectively). Vasectomy was significantly increased from 1 in the first year to 39 in the second year ( $p$-value, $0.4 \%$ ).

In 2017, Fauzia et al. in their study showed that the most widely used method was OCPs (27\%). It was followed by injectables (12.4\%), condoms (6.4\%), female sterilization (4.6\%), male sterilization (1.2\%), implants (1.7\%), and IUDs $(0.6 \%)^{1}$

Among currently married couples, $8 \%$ use LARC, such as implants or IUCDs, or a permanent method of contraception, such as female or male sterilization. Use of long-acting and permanent methods raised at 30\% in 1991 but declined steadily, and now, the current rate is $8 \%$ in $2007 .{ }^{17}$ Susan et al. in their study in rural Kenya showed in an interview of 423 contraception users, $20.6 \%(95 \% \mathrm{Cl}$, 17-25\%) utilized LARC methods, while $79.4 \%$ (336) chose the shortterm methods. The use of the different methods was reduced as follows: Injectables, 253 (59.8\%); pills, 83 (19.6\%); implants, 71 (16.8\%); and IUCDs, 16 (3.8\%). No client showed interest for voluntary surgical contraception during the study period. ${ }^{18}$

All these studies go similar to our study results that there is more uptake of short-term contraception than LARC. We could not interview patients, but according to family planning workers, weight gain, irregular bleeding, and issues about insertion and removal of implant and IUCD were the main causes of less uptake of and discontinuation of LARC. Table 2 shows that misoprostol and MM kit-induced abortion were increased significantly in $\mathrm{CMH}$ of Bogura, TMSS, and MSC. SHC had only outpatient service. From a casual interview of the treating physicians of MSC and TMSS and our experience from $\mathrm{CMH}$ of Bogura, all of these pregnancy complications were due to taking misoprostol or MM kit for secret or unintended abortion, without any doctor's prescription, in wrong gestational age and inappropriate dose. They also inform that most of these patients were not on any method of contraception or on an irregular pill or condom use. Clandestine abortion is always been a serious health problem in Bangladesh. Anecdoctal reports show that the secret use of misoprostol has increased since the

Table 1: Comparison of consummation of contraceptive methods in observed organizations

\begin{tabular}{|c|c|c|c|c|c|c|c|}
\hline \multicolumn{8}{|c|}{ CMH of Bogura } \\
\hline Year & IUCD & Implant & Injectables & $O C P$ & Condoms & Tubectomy & Vasectomy \\
\hline 2017-2018 & $14(0.00081 \%)$ & 0 & $282(0.16 \%)$ & $10,387(6 \%)$ & $162,800(93 \%)$ & $58(0.03 \%)$ & 0 \\
\hline 2018-2019 & 01 (0.0005\%) & 0 & $271(0.13 \%)$ & $12,036(6 \%)$ & $184,100(93 \%)$ & $45(0.003 \%)$ & 0 \\
\hline$p$-value & 0.0001 & 0 & 0.13 & 0.047 & 0.039 & 0.08 & 0 \\
\hline \multicolumn{8}{|l|}{ TMSS } \\
\hline 2017-2018 & $23(0.2 \%)$ & $103(0.95 \%)$ & 330 (3.07\%) & $5,534(51.5 \%)$ & $4,589(42.7 \%)$ & $32(0.36 \%)$ & 0 \\
\hline 2018-2019 & $30(0.2 \%)$ & $249(2.1 \%)$ & $288(2.4 \%)$ & $5,672(48.1 \%)$ & $5,672(43.4 \%)$ & $68(0.5 \%)$ & 0 \\
\hline$p$-value & 0.084 & 0.063 & 0.043 & 0.104 & 0.035 & 0.22 & 0 \\
\hline \multicolumn{8}{|l|}{ SHC } \\
\hline 2017-2018 & 19 (0.12\%) & $75(0.5 \%)$ & 4,289 (29\%) & $6,820(46 \%)$ & $3,411(23 \%)$ & $17(0.1 \%)$ & 0 \\
\hline 2018-2019 & $13(0.08 \%)$ & $72(0.4 \%)$ & $4,226(28 \%)$ & $4,885(38 \%)$ & $5,679(38 \%)$ & $20(0.1 \%)$ & 0 \\
\hline$p$-value & 0.118 & 0.25 & 0.226 & 0.008 & 0.156 & 0.5 & 0 \\
\hline \multicolumn{8}{|l|}{ MSC } \\
\hline 2017-2018 & 284 (1.8\%) & 545 (3.5\%) & 237 (1.5\%) & $2,118(13 \%)$ & $11,760(76 \%)$ & $0(0 \%)$ & $1(0.4 \%)$ \\
\hline 2018-2019 & $285(1.3 \%)$ & $516(2.4 \%)$ & $516(2.4 \%)$ & 3,027 (14\%) & $15,980(76.2 \% 5)$ & $3(0.05 \%)$ & $39(0.18 \%)$ \\
\hline$p$-value & 0.445 & 0.71 & 0.005 & 0.01 & 0.096 & 0.5 & 0.4 \\
\hline
\end{tabular}


Table 2: Comparison of MM kit or misoprostol-induced abortion with complications in observed organizations

\begin{tabular}{llll}
\hline Year/institution & CMH of Bogura & TMSS & MSC \\
\hline $2017-2018$ & $27(0.0165 \%)$ & $128(1.19 \%)$ & $419(2.7 \%)$ \\
$2018-2019$ & $55(0.028 \%)$ & $360(3.05 \%)$ & $581(2.77 \%)$ \\
$p$-value & 0.02 & 0.05 & 0.01 \\
\hline
\end{tabular}

most recent estimates (from 2010). A study conducted in India on incomplete abortion after medical abortion on 100 women showed $30 \%$ of incomplete abortion in the center after medical abortion. ${ }^{19}$

\section{Discussion}

This study showed that 15 to 45 years was the age-group of women who were aware of varieties of contraception. OCPs and condoms were most well-known and used among consumers. In the last four decades, the CPR has increased. But inconsistent and incorrect use and frequent switching it are counterbalanced by discontinuation, switching, and incorrect use with a steady decline in the use of longacting and permanent methods. So, estimating its long-term poor health impact on women's health, in the new Health Population and Nutrition Sector Development Program (HPNSDP) (2011-2016), the government has taken elaborate measures to promote the use of long-acting and permanent methods. ${ }^{20}$ Here, in this study, we can see that the use of LARC is static or declining in most of the centers. More use of LARC has been observed in TMSS. We went there and found that the family planning officer was very expert and experienced. She informed that women are less interested in IUCD for fear of insertion, removal, and irregular bleeding. They denied injectables due to weight gain, irregular bleeding, and amenorrhea. For the reason of insertion of the implant, they also were less interested in using it. According to her, education level is a factor for uptake of LARC. Proper and thorough and prolonged counseling is also required. This coincides with a study done by Ontiri et al. ${ }^{18}$ In their study, after a casual interview with family planning officers, it was assumed that for counseling a client about LARC, it takes 15 to 20 minutes, whereas that for short-term method, it takes only 5 minutes. It saves time and reduces the waiting period of the clients. It was also shown that women who take LARC were willing to discontinue it influenced by their neighbors or other users. Some women are less interested to take IUCD as their husbands will feel it during intercourse. Rather they prefer OCP or injections instead of LARC. Lack of training of insertion and removal of IUCD and implant was also reflected. As there are less clients to use it, new trainees get less chance to learn.

The 2014 Kenya Demographic Health Survey findings, released in 2015, confirmed that the prevalence of short-term method users (63.6\%) was higher than the LARC method (25.0\%) among modern contraceptive users in Kakamega County. ${ }^{18,21}$

In our study, tubectomy has reduced in the second year in $\mathrm{CMH}$ of Bogura, MSC, and SHC, but is increased in TMSS. Male sterilization was absent in three centers except for MSC. The use of LARC and female sterilization has been reduced in the last two decades. Higher use of short-term methods (OCP and injectables) and female sterilization, compared to a lower rate of use of condoms and male sterilization, resembles that males are more reluctant in taking or maintaining a good contraceptive practice as well as male domination in society. The objective of our study was to observe the trend of contraception uptake, acceptability of LARC, and to see its drawbacks such as inappropriate and inconsistent use of contraception leading to unintended pregnancy. People are using misoprostol and buying MM kit from drug sellers without any doctor's prescription. They are considering this as an alternative to contraception unknowingly their harmful effects that may endanger their lives. Most of these patients had no contraception history or they were on OCP or barrier irregularly. Using single dose of MM kit or misoprostol compels them to think that this is less cumbersome than taking regular contraceptive pills or wearing IUCD or implants. All of them took misoprostol or MM kit as advised by their neighbors and nonprofessionals, in the wrong dose, incomplete dose, and unsupervised. In our study, in all the three centers, induced abortion with medical method with complications increased significantly. Complications were incomplete abortion, sepsis, and hemorrhage. According to Henderson, although excessive bleeding was followed by medical termination, the requirement for blood transfusion was low. ${ }^{22}$ After a medical abortion, the approximate frequency of diagnosed or treated infection is low and varies according to the regimens used. Also, it is not life-threatening. But Anuya et al..$^{19}$ in their study showed $63 \%$ of patients following medical method of abortion (MMA) required blood transfusion. No maternal mortality was seen in our study in any center.

\section{Limitations}

Our study limitations were as follows:

- The sample did not exactly represent all women of reproductive age, since it only consisted of women receiving a FP service from public health facilities, we intended to collect data from government health facilities where contraceptive commodities are provided free of charge.

- It would be better if we could interview the clients so better evaluation and more information about method mix, method switching, and discontinuation could be obtained.

\section{ConClusion}

According to our study, LARC uptake is reduced overall. Short-term method is more preferred. Female sterilization has no significant change. Male sterilization is very less in number. People are not adherent to contraception, resulting in a more accidental pregnancy. OTC availability of abortifacients makes these patients to take them as a quick remedy. This is resulting in increased health hazards for women such as abortion-related complications. So, a program should be implemented to increase the use of LARC uptake. This can be done by increasing the number of stuff, arranging training programs about LARC services, improving contraception counseling, and increasing male participation in family planning programs. Moreover, OTC or self-use of MM kit should be avoided. Doctors as well as the women using MM should be well aware of the possible side effects, amount of bleeding which is alarming, and when to report back to the hospital. Education has a great role to help women to choose contraceptive methods and use abortion services when needed. Future studies should focus on the sociodemographic and reproductive health determinants that change with the increased adoption of LARC methods among contraceptive users.

\section{Recommendations}

- LARC should have a stronger campaign.

- More and more family planning workers should be trained on LARC 
- OTC selling of abortifacients should be restricted

- Campaign against unsupervised use of misoprostol should be started.

\section{References}

1. Greenspan A. Fertility decline in Bangladesh: an emerging family planning success story. Asia Pac Popul Policy 1992;20:1-4. PMID: 12285494

2. Huda FA, Robertson Y, Chowdhuri S, et al. Contraceptive practices among married women of reproductive age in Bangladesh: a review of the evidence. Reprod Health 2017;14(1)69. DOI: 10.1186/s12978-017-0333-2.

3. Bairagi $R$, Islam M, Barua MK. Contraceptive failure: levels, trends and determinants in Matlab, Bangladesh. J Biol Sci 2000;32(1):107-123. DOI: $10.1017 / \mathrm{s} 0021932000001073$.

4. Blumenthal P, Voedisch A, Gemzell-Danielsson K. Strategies to prevent unintended pregnancy: increasing use of long-acting reversible contraception. Hum Reprod 2011;17(1):121-137. DOI: 10.1093/humupd/dmq026.

5. Trussell J, Guthrie K. Choosing a contraceptive: efficacy, safety, and personal considerations. In: Hatcher RA, Trussell J, Nelson AL, et al., editors. Contraceptive technology. 19th ed. New York, NY, USA: Ardent Media, Inc.; 2007. p. 19-47.

6. Jacobstein R, Stanley H. Contraceptive implants: providing better choice to meet growing family planning demand. Glob Health Sci Pract 2013;1(1):11-17. DOI: 10.9745/GHSP-D-12-00003.

7. Bangladesh Demographic and Health Survey 2014. National Institute of Population Research and Training (NIPORT). Dhaka: Ministry of Health and Family Welfare; 2015.

8. Adolescent Health Fact Sheet. WHO. 2007.

9. Hossain A, Maddow-Zimet I, Ingerick M, et al. Access to and quality of menstrual regulation services and postabortion care in Bangladesh, 2014, New York: Guttmacher Institute; 2017.

10. The medical termination of pregnancy amendment act, 2002. No. 62 of 2002, 18th December 2002.

11. Comprehensive abortion care training and service delivery guideline. Ministry of Health and Family Welfare Government of India; 2010.
12. Mundle S, Elul B, Anand A, et al. Increasing access to safe abortion services in rural India: experiences with medical abortion in a primary health centre. Contraception 2007;6:66-70. DOI: 10.1016/j. contraception.2007.03.010.

13. Coyaji K, Elul B, Krishna U, et al. Mifepristone-misoprostol abortion: a trial in rural and urban Maharashtra, India. Contraception 2002;66:3340. DOI: 10.1016/S0010-7824(02)00309-8.

14. Kopp Kallner H, Fiala C, Stephansson O, et al. Home self-administration of vaginal misoprostol for medical abortion at 50-63 days compared with gestation of below 50 days. Hum Reprod 2010;25:1153-1157. DOI: 10.1093/humrep/deq037.

15. Kahn JG, Becker BJ, Maclssac L, et al. The efficacy of medical abortion: a meta-analysis statistics. Standford.edu/ckirby/techrepots/ GEN/1999/1999-29.

16. Lara D, Garcia SG, Wilson KS, et al. How often and under which circumstances do Mexican Pharmacy vendors recommend misoprostol to induce an abortion? Int Perspect Sex Reprod Health 2011;37:75-83. DOI: 10.1363/3707511.

17. Bangladesh Demographic and Health Survey 2007. National Institute of Population Research and Training (NIPORT), Dhaka, Bangladesh; 2009.

18. Ontiri $S$, Ndirangu $G$, Kabue $M$, et al. Long-acting reversible contraception uptake and associated factors among women of reproductive age in rural Kenya. Int J Environ Res Public Health 2019;16(9):1543. DOI: 10.3390/ijerph16091543.

19. Singh S, Hossain A, Zimet IM, et al. The incidence of menstrual regulation procedures and abortion in Bangladesh, 2014. Int Perspect Sex Reprod Health 2017;43(1):1-11. DOI: 10.1363/43e2417.

20. Health, Population, and Nutrition Sector Development Program (2011-2016), Program Implementation Plan. In: MoH \& FW, editor. Dhaka: Government of Bangladesh.

21. Kenya National Bureau of Statistics. Ministry of Health/Kenya. National AIDSControl Council/Kenya. Kenya Medical Research Institute. National Council for Population Development/Kenya. Kenya demographic and health survey 2014. Rockville, MD, USA: ICF Macro; 2015.

22. Shenon C, Perry Brothers L, Philip NM, et al. Infection after medical abortion: a review of literature. Contraception 2004;70(3):183-190. DOI: 10.1016/j.contraception.2004.04.009. 